Ann. Biol. anim. Bioch. Biophys., I975, 15 (4), 745-750.

\title{
ACTION DE L'HYDROXYDE DE POTASSIUM SUR LES CHROMOSOMES HUMAINS
}

\author{
R. BERGER \\ Centre de Recherches biologiques néonatales, \\ Hopital Port-Royal, \\ 123, Bd de Port-Royal, \\ 75014 Paris \\ RÉSUMÉ
}

L'effet de l'hydroxyde de potassium sur les chromosomes humains a été étudié. Trois sortes d'aspects peuvent être observés : aspect en "pointillé " des chromatides qui pourrait correspondre à un aspect particulier correspondant à la structure en spirale des chromosomes; bandes G; aspect partiellement inverse de celui des bandes C. La localisation des bandes inverses des bandes $\mathrm{C}$ dans certaines régions centromériques et à certaines constrictions secondaires suggère que l'hydroxyde de potassium agit principalement sur le DNA satellite, et particulièrement sur le DNAs II.

L'hydroxyde de sodium a été utilisé dans les premières techniques d'obtention des bandes $\mathrm{C}$ et $\mathrm{G}$ avec coloration par le Giemsa (Arrighr et Hsu, I97I ; DrETs et Shaw, I97I ; Schnedl, I97I). Sumner et al. (I97I) ont montré que l'emploi de la soude n'était pas nécessaire à l'obtention des bandes G. A l'inverse nous avons obtenu des bandes $G$ en tutilisant l'hydroxyde de sodium seul, avant coloration par le Giemsa (BERGER, I972). Nous avons ensuite étudié de façon systématique l'effet de l'hydroxyde de potassium sur les chromosomes humains afin de le comparer à celui de l'hydroxyde de potassium.

\section{MATÉRIEL，ET MÉTHODES}

Les chromosomes ont été préparés à partir de cultures de sang provenant de 25 sujets des deux sexes examinés à divers titres. Ces cultures ont été faites selon notre microméthode habituelle (solution hypotonique : $\mathrm{KCl} 0,075 \mathrm{M}$; fixation avec le mélange éthanol-acide acétique dans les proportions $3: \mathrm{I}$ ). Les solutions d'hydroxyde de potassium sont préparées juste avant 
l'emploi, à des concentrations variant entre $0,0002 \mathrm{M}$ et $0,00 \mathrm{IM}$. Les lames utilisées, préparées à des intervalles de temps divers, compris entre quelques heures et quclques jours, sont plongées à température ambiante dans la solution d'hydroxyde de potassium pendant une durée allant de vingt secondes à io minutes. Elles sont ensuite rincées à l'eau courante et plongées dans une solution de Giemsa à 4 p. Ioo tamponnée à $\mathrm{pH} 6,7$.

\section{RÉSULTATS}

L'action de l'hydroxyde de potassium sur les chtomosomes donne plusieurs sortes de résultats. Il nous est apparu que les aspects les plus intéressants ont été obtenus à la concentration de $0,0005 \mathrm{M}$, avec des lames préparées la veille de la manipulation. Les effets sont moins nets avec des lames plus anciennes, et ils sont difficiles à contrôler avec des lames préparées dans la même journée. Le temps d'action optimum dans ces conditions varie quelque peu selon les sujets et selon le résultat désiré. Il est compris en moyenne entre 3 et 6 minutes.

On peut distinguer trois sortes de résultats selon la durée d'action de la solution de potasse, soit, en allant du temps le plus bref au temps le plus long : un aspect en " pointillé ", un " marquage " en bandes G, un aspect paraissant inverse de celui des bandes C (P1. I, fig. I).

On peut en effet observer sur certaines préparations une série de points de dimension identique sur chaque chromosome et régulièrement espacés sur les deux chromatides sœurs, donnant l'impression d'un pointillé régulier. Le nombre de points varie avec le degré de contraction des chromosomes ; ils sont d'autant plus nombreux que les chromosomes sont moins contractés et que la longueur de l'élément est plus grande. La distribution des points est le plus souvent identique sur les deux chromatides d'un chromosome métaphasique. Il est à remarquer que le chromosome $n^{\circ} \mathbf{I}_{7}$ réagit particulièrement avec l'hydroxyde de potassium. L'aspect en " pointillé " peut parfois être rendu plus manifeste après coloration par l'orangé d'acridine et examen en lumière ultraviolette.

Un aspect de bandes $\mathrm{G}$ peut également être obtenu avec cette méthode. Ces bandes sont tout à fait comparables à celles qui sont obtenues avec d'autres techniques de bandes $\mathrm{G}$ (Paris Conference, I97I). Il n'est pas exceptionnel d'observer dans une même cellule un aspect intermédiaire entre celui des "pointillés " et celui des bandes $G$, certains chromosomes étant marqués en bandes $G$, d'autres en pointillé.

Avec les temps d'action plus long de la solution de potasse, les chromosomes ont un aspect différent : les régions proches du centromère et certaines constrictions secondaires sont pâles, peu colorées, donnant une apparence en quelque sorte inverse de celle des bandes C. La distribution de ces zones peu colorées par le Giemsa n'est cependant pas entièrement superposable à celle des bandes $C$ telle qu'elle a été cléfinie à la Conférence de Paris (I97I). I.es différences essentielles portent sur la présence de zones peu colorées sur les chromosomes no 2 , sur l'un des nos $4-5$, sur deux éléments du groupes $C$ (en plus du $n^{\circ}$ ), sur un des chromosomes du groupe $\mathrm{D}$, le $\mathrm{I} 6$ étant le plus souvent moins " marqué " qu'avec les techniques des bandes C (P1. I, fig. 2).

L'aspect du chromosome $\mathrm{Y}$ est particulier chez certaines personnes : il existe 
à l'extrémité du bras long une zone intensément colorée par le Giemsa, mais moins étendue que celle que l'on observe avec la technique des bandes $\mathrm{C}$ ou la coloration avec les dérivés de la quinacrine. On a l'impression dans ces cas qu'il existe deux régions différentes à l'extrémité du bras long de l' $Y$, une zone intensément colorée et une zone plus pâle, plus colorée que le reste du chromosome.

\section{DISCUSSION}

L'effet des bases sur les chromosomes de hamster chinois a été étudié par Kato et Moriwaki (I972) qui ont ainsi obtenu des bandes et signalé l'effet brutal qu'elles peuvent avoir sur les préparations microscopiques.

L'aspect " en pointillé " est difficile à interpréter actuellement avec certitude. La taille identique de chacun des points sur tous les chromosomes d'une même cellule, leur distribution régulière le long des chromatides et leur concordance entre chromatides sœurs, suggèrent qu'il peut s'agir d'un aspect particulier de la spiralisation des chromosomes. Cette interprétation est rendue d'autant plus plausible que dans certaines cellules un aspect en spirale proche de celui décrit par OHNucki (I968) et RUZICKA (I973) peut être observé. Il est cependant difficile de faire un décompte précis des points puisque leur nombre varie avec le degré d'élongation des chromosomes. Cette étude quantitative serait entachée d'une marge d'erreur non négligeable, comme le montrent les travaux des deux auteurs mentionnés ci-dessus, qui ont étudié cette spiralisation avec d'autres méthodes.

L'obtention des bandes $G$ avec 1'hydroxyde de potassium nous avait d'abord paru pouvoir être due à un effet sur les protéines acides constitutives des chromosomes. L'interprétation du rôle des techniques de production des bandes $\mathrm{G}$ doit en fait être reconsidérée car on peut obtenir ces bandes sans autre préparation que l'emploi de colorant de Giemsa dilué (TAKAYAMa, I974; WALTHER et al., I974). Les bandes $\mathrm{G}$ correspondent donc vraisemblablement à une structure prééxistante des chromosomes, les différentes techniques de production de bandes $G$ ne les induisant pas, mais favorisant seulement leur révélation.

L'aspect particulier des régions centromériques et des constrictions secondaires obtenu après action de 1'hydroxyde de potassium mérite d'être discuté. La localisation même des zones per colorées dans ces régions et sa similitude partielle avec celle des bandes $\mathrm{C}$ fait penser que la potasse a agi sur 1'hétérochromatine correspondant au DNA satellite. On peut distinguer au moins quatre sorte de DNA satellite qui se différencient par certaines caractéristiques biochimiques, en particulier les densités appréciées par sédimentation en chlorure de césium alcalin (CORNEO et al., I973). La localisation des DNA satellite I, II et III sur les chromosomes humains est connue (JONEs et CorNEO, I97 I ; JonEs et coll., I973 et I974), et celle du DNAs IV l'est en partie (SAUNDERS et coll. I972; EvANs et al., I974). Elle correspond à peu près à celle des bandes $\mathrm{C}$. Or il existe des différences entre la localisation des bandes $\mathrm{C}$ et celle des régions peu colorées après action de l'hydroxyde de potassium. Ces différences portent avant tout sur le chromosome 2, en général peu marqué avec la technique des bandes $\mathrm{C}$, et qui contient du DNAs II et III. Les chromosomes 4 et 5 diffèrent par le fait que le premier contient du DNAs I, II et III 
alors que le second contient du INAs I et III. Fnfin les chromosomes 7 et ro contiennent du DNAs II. On peut donc penser que l'hydroxyde de potassium agit préférentiellement sur le DNAs II, plus que sur le DNAs III et surtout I. Ainsi s'expliquerait également l'aspect particulier du chromosome $\mathrm{Y}$, dont on sait qu'il contient les 4 sortes de DNA satellite. Il n'est d'ailleurs pas impossible que le DNAs IV soit aussi plus réactif que le DNAs I vis-à-vis de l'hydroxyde de potassium. Dans cette hypothèse le chromosome II pourrait en contenir.

Colloque D. G. R. S.T., Biologie de la Procréation, Paris, $7-8$ mars 1975 .

\section{REMERCIEMENTS}

Travail effectué avec l'aide de la D. G. R. S. T., contrat no 72-7-0376.

\section{SUMMARY}

\section{EFFECT OF POTASSIUM HYDROXIDE ON HUMAN CHROMOSOMES}

The effect of potassium hydroxide on human chromosomes was studied. Three kinds of patterns were observed : dotted appearance which could be a particular aspect of figure of chromosomes spiral structure, $\mathrm{G}$ bands, and partially reverse $\mathrm{C}$-bands. The location of reverse $\mathrm{C}$ bands in the centromere and secondary constrictions regions suggests that potassium hydroxide acts mainly on DNA satellite, and mainly on DNAs II.

\section{RÉFÉRENCES BIBLIOGRAPHIQUES}

Arright F. E., Hsu T. C., I971. Localization of heterochromatin in human chromosomes. Cytogenetics, 10, $8 \mathrm{I}-86$.

Berger R., ig72. Effet de l'hydroxyde de sodium sur les chromosomes. C. R. Acad. Sc. (Paris), 275, I5II-I5I2.

Corneo G., Ginelli E., Zardi L., I973. Satellite and repeated sequences in human DNA, 29-37, in : Modern Aspects of Cytogenetics : Constitutive Heterochromatin in Man.Symposia Medica Hoechst 6, ed. by R. A. Pfeifrer ; Schattauer, Stuttgart.

Drets M. E., Shaw M. W., i97i. Specific banding patterns of human chromosomes. Proc. Nat. Acad. Sci. U. S. A., 68, 2073-2077.

Evans H. J., Gosden J. R., Mrtcinll A. R., Buckland IR. A., I974. Location of human satellite DNAs on the Y chromosome. Nature, 251, 346-347.

Jones K. W., Corneo G., I97I. Location of satellite and homogeneous DNA sequences on human chromosomes. Nature New Biol., 233, 268-271.

Jones K. W., Prosser J., Corneo G., Ginelli E., i973. The chromosomal location of human satellite DNA III. Chromosoma, 42, 445-45I.

Jones K. W., Purdom I. F., Prossfr J., Corneo (i., I974. The chromosomal localisation of human satellite DNA I. Chromosoma, 49, I6I-I7I.

Kato H., Moriwaki K., I972. Factors involved in the production of banded structures in mammalian chromosomes. Chromosoma, 38, 105-Izo.

OHNucki Y., I968. Structure of chromosomes. I. Norphological studies of the spiral structure of human somatic chromosomes. Chromosoma, 25, 4 o2-428. 
PARIS CONFERLNCE, 197I. Standardization in human cytogenetics. Birth Defects : Original Article Series VIII, 7. The National Foundation, New York, 1972.

RuzıckA F., r973. Über die Primriwindungen menschlicher Chromosonten. Humangenetik, 20, 33534 .

Saunders G. F., Hsv T. C., Get\% M. J., Sines E. L., Arrighi F, E, I972. Jocations of a human satellite DNA in human chromosomes. Nature Neto Biol., 236, 244-246.

Schnedt. W., ig7r. Analysis of the human karyotype using a reassociation technique. Chromosoma, 34, $44^{8-454}$.

Sumner A. T., Evans H. J., Buckland R. A., I97I. New technique for distinguishing between human chromosomes. Nature New Biol., 232, 3I-32.

TAKAYAMA S., 1974. Factors for (riemsa-band formation in air-dried mammalian chromosomes. Japan. J. Genet., 49, 189-I95.

Walther J. U., Stengel-Rutkowski S., Murken J. D., 1974. Observations with G banding of human chromosomes. Humangenetik, 25, 49-5i. 


\section{PLANCHE I}

Chromosomes $\mathrm{n}^{\circ} \mathrm{I}, \mathrm{I} 7$ et $\mathrm{Y}$. $a$, aspect en " pointillé $" ; b$, aspect intermédiaire entre "pointillé " et en spirale ; $c$, bandes $\mathrm{G}$; $d$, aspect inverse des bandes $\mathrm{C}$. Chromosome $\mathrm{Y}$; comparaison avec l'aspect des bandes $Q$. 


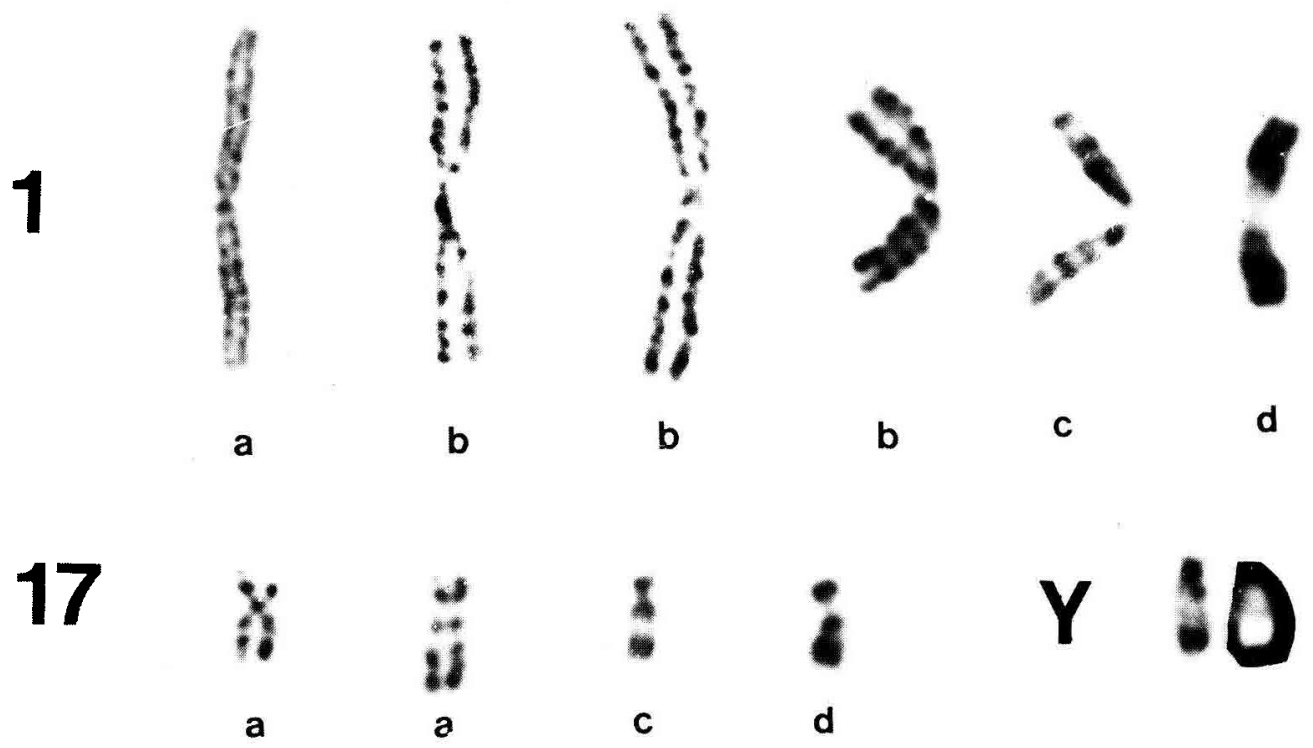




\section{PLANCHE II}

Comparaison entre bandes $\mathrm{C}$ et inverse des bandes $\mathrm{C}$. 

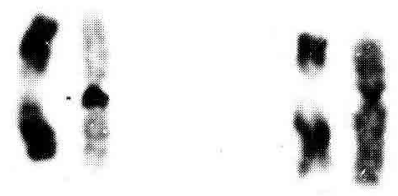

울

6

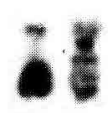

10

$2 \%$

13

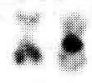

16

.8 .9

19

4. 4

21
2

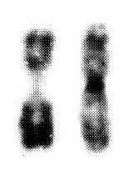

3

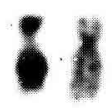

8

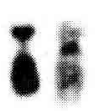

12

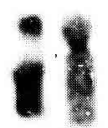

4

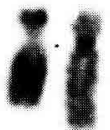

5
$: 1$

9

:1

x

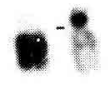

15

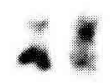

17

2.

20

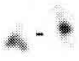

22

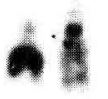

14

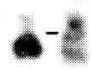

18

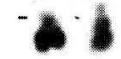

$Y$ 